\title{
High Resolution Studies of Oxide Multiferroic Interfaces in the Aberration- Corrected STEM
}

Javier Grandal $^{1}$, Juan I. Beltran ${ }^{1}$, Gabriel Sanchez-Santolino ${ }^{2}$, Fernando Gallego ${ }^{3}$, Javier Tornos ${ }^{3}$, Mariona Cabero ${ }^{1}$, Carlos Leon ${ }^{1,4}$, Federico Mompean ${ }^{3}$, Mar García-Hernández ${ }^{3}$, Stephen J. Pennycook ${ }^{5}$, M. Carmen Muñoz ${ }^{3}$, Jacobo Santamaria ${ }^{1,4}$ and Maria Varela ${ }^{1,4}$

1. GFMC, Dept. de Fisica de Materiales, Universidad Complutense de Madrid, 28040 Madrid, Spain.

2. University of Tokyo, Tokyo 113-8656, Japan.

3. Instituto de Ciencia de Materiales de Madrid, Consejo Superior de Investigaciones Científicas. Cantoblanco 280409, Madrid. Spain.

4. Instituto de Magnetismo Aplicado, Universidad Complutense de Madrid, 28040 Madrid, Spain.

5. Department of Materials Science \& Engineering, National University of Singapore, Singapore 117575.

Complex oxides are challenging systems where subtle changes in structure, electronic doping or chemistry may result in colossal responses in macroscopic physical behavior. Amongst complex oxides we can find insulators, high-Tc superconductors, ferromagnets, ferroelectrics, multiferroics and a wide list of families displaying the most disparate physical behaviors. Many of these materials exhibit a perovskite-like structure. Being highly compatible with each other, high quality epitaxial heterostructures can be grown where novel macroscopic functionalities arise. This is the case when oxide materials exhibiting different ferroic orders are combined. The robustness of such multiferroic heterostructures makes them very appealing from the point of view of technological applications. One factor that strongly influences the properties of such layered systems is the stoichiometry of the constituent ultrathin films. Unfortunately, the actual film composition is often difficult to establish. Even more so when the physics of the system may be controlled by small concentrations of oxygen vacancies. Small densities of these point defects can often explain observed phenomena in oxide heterostructures and documenting their existence has been a challenge that remains under dispute.

In this talk we will discuss the electronic and magnetic properties of oxide based ferromagnetic /ferroelectric interfaces where titanate layers such as ultrathin ferroelectric $\mathrm{BaTiO}_{3}$ (BTO) barriers are sandwiched between ferromagnetic $\mathrm{La}_{0.7} \mathrm{Sr}_{0.3} \mathrm{MnO}_{3}$ (LSMO) electrodes in high quality LSMO/BTO/LSMO trilayers grown by high-pressure $\mathrm{O}_{2}$ sputtering. We will combine the use of advanced electron microscopy techniques such as electron energy-loss spectroscopy (EELS) in the aberration-corrected scanning transmission electron microscope (STEM) with density-functional calculations to study any local electronic phenomena giving rise to coupling across the interfaces and, hence, multiferroic behavior. Real space measurements of local polarization will be compared to magnetic quantities inferred from energy-loss magnetic chiral dichroism (EMCD), a technique directly sensitive to the local magnetic moment. High spatial resolution EMCD measurements obtained at low temperature $(<100 \mathrm{~K})$ allow studying the local magnetization of the interfaces in the sub-nm scale [1, 2], see Figure 1. On the other hand, the quantification of local ferroelectric polarization is possible through the analysis of atomic resolution annular bright field images [3]. Both local magnetization and polarization measurements will be discussed in the light of electronic properties such as interface charge transfer and orbital anisotropy through density-functional theory calculations. 
References:

[1] P. Schattschneider et al, Nature 441 (2006) pp. 486-488.

[2] J. Salafranca et al, Nanoletters 12 (2012), pp. 2499-2503.

[3] G. Sanchez-Santolino et al, Nature Nanotechnolgy, accepted (2017).

[4] Research at UCM sponsored by Fundación BBVA and Spanish MINECO/FEDER MAT201566888-C3-1-R \& MAT2015-66888-C3-3-R and by the ERC Proof of Concept Grant MAGTOOLS.
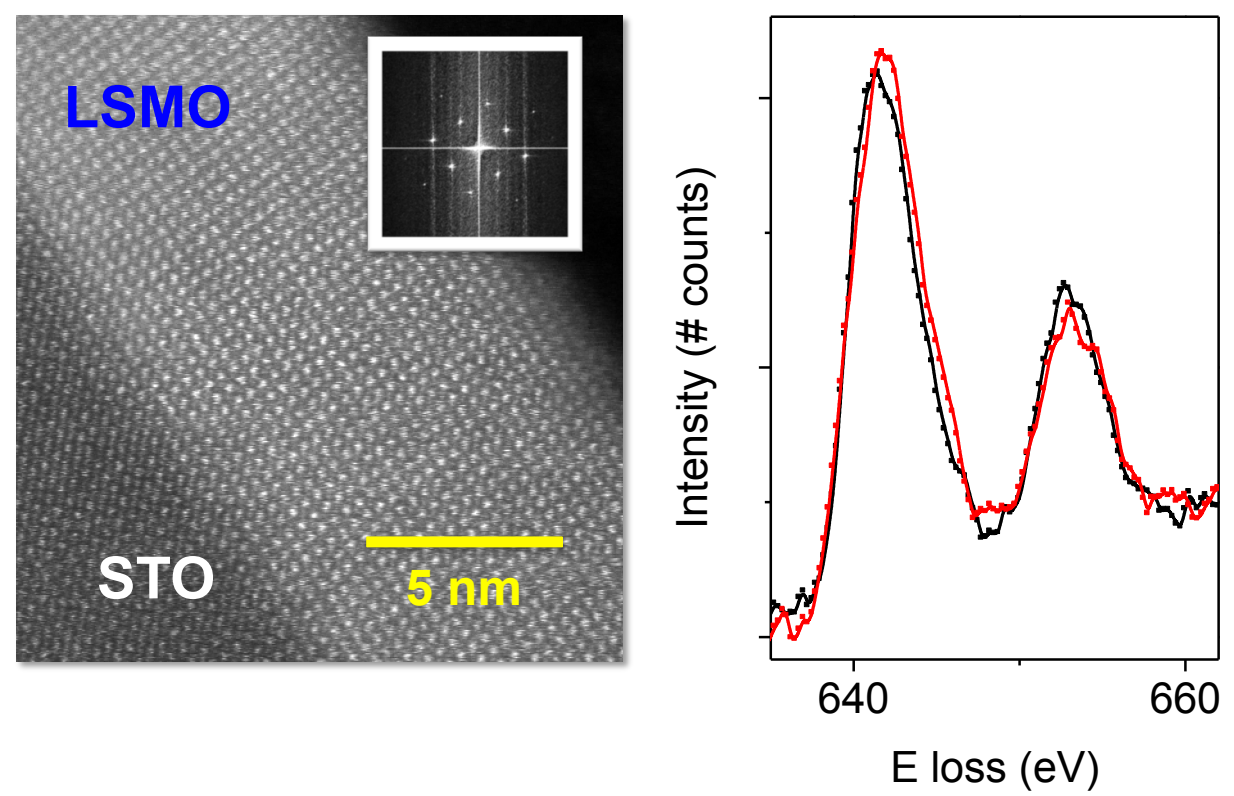

Figure 1. (left) Atomic resolution image of a $\mathrm{SrTiO}_{3} / \mathrm{LSMO}$ interface acquired at $98 \mathrm{~K}$. While some noise is visible, the specimen stability is noticeable. The inset exhibits the Fourier transform of the image. (Right) $M n L_{2,3}$ edges obtained in nanodiffraction mode using an approximate convergence angle of $15 \mathrm{mrad}$. They were acquired by effectively placing the aperture in two different conjugate positions in the nanodiffraction pattern. Despites noise, a clear dichroic signal is observed which can be tracked across the interface to study the magnetic properties with high spatial resolution. Data acquired in an aberration corrected JEOL ARM200cF operated at $200 \mathrm{kV}$. 